\title{
Interfacial free energy and stiffness of aluminum during rapid solidification
}

\author{
Nicholas T. Brown ${ }^{\mathrm{a}, \mathrm{b}}$, Enrique Martinez ${ }^{\mathrm{b}}$, Jianmin $\mathrm{Qu}^{\mathrm{a}}$ \\ ${ }^{a}$ Northwestern University; 2145 Sheridan Road, Room B224, Evanston, IL 60208 \\ ${ }^{b}$ Los Alamos National Laboratory; MST-8, Materials Science \& Technology, Los Alamos, NM 87544
}

\begin{abstract}
Using molecular dynamics simulations and the capillary fluctuation method, we have calculated the anisotropic crystal-melt interfacial free energy and stiffness of aluminum in a rapid solidification system where a temperature gradient is applied to enforce thermal non-equilibrium. To calculate these material properties, the standard capillary fluctuation method typically used for systems in equilibrium has been modified to incorporate a second-order Taylor expansion of the interfacial free energy term. The result is a robust method for calculating interfacial energy, stiffness and anisotropy as a function of temperature gradient using the fluctuations in the defined interface height. This work includes the calculation of interface characteristics for temperature gradients ranging from $11 \sim 34 \mathrm{~K} / \mathrm{nm}$. The captured results are compared to a thermal equilibrium case using the same model and simulation technique with a zero gradient definition. We define the temperature gradient as the change in temperature over height perpendicular to the crystal-melt interface. The gradients are applied in MD simulations using defined thermostat regions on a stable solid-liquid interface initially in thermal equilibrium. The results of this work show that the interfacial stiffness and free energy for aluminum are dependent on the magnitude of the temperature gradient, however the anisotropic parametersremainindependent of the nonequilibrium conditionsapplied in this analysis. The relationships of the interfacial free energy/stiffness are determined to be linearly related to the thermal gradient, and can be interpolated to find material characteristics at additional temperature gradients.
\end{abstract}

Keywords: rapid solidification; molecular dynamics; solid-liquid interface, interfacial stiffness; interface free energy

\section{Introduction}

The study of rapid solidification through computational analyseshas seen an increase in demanddue to the recent advances in the additive manufacturing industry. These processes apply extreme temperature gradientsto propagate the interface between the solid and liquid phases of the materialto form a new microstructure. The extreme environmental impacts on the material often result in the development of intrinsic defects that contribute to the degradation of the material strengthand limit the applications of these structural components[1-4].For standard solidification phenomena, multi-scale simulations provide us with a means to study the formation of these microstructures at an atomic scale, as well as the progression and impact of these defects through phase-field and finite element method simulations[5-9]. When these theories are applied to rapid solidification processes, the assumption of thermal equilibrium may not result in the most accurate representation of the physical state[10-13].This paper focuses on the atomic scale of the rapid solidification of aluminum through molecular dynamicswith the application of the capillary fluctuation method and an applied thermal gradient that drives the system out of equilibrium to capture the changes in the interface characteristics.

The strength of metallic structures formed through solidification processes is largely affected by the growth patterns of microstructures known as dendrites. These crystalline structures arethe result of a moving solid-liquid interface and are constrained by thefluctuating 
heating and cooling temperatures[7, 11, 14-17]. For standard solidification processes, such as welding, the geometric growth patterns of these dendrites have been shown to be dependent primarily on the individual tip velocity and interfacial stiffness for the material[5, 18]. These patterns are typically studied through phase-field simulations using an equilibrium-based GibbsThomson condition, oftensupplemented with results from molecular dynamic (MD) simulations. However, for rapid solidification processes, where the heating and cooling rates can be on the order of $10^{3}-10^{8} \mathrm{~K} / \mathrm{s}[19]$, the structures are formed through extreme thermodynamic environments forcing the crystal melt interface into a non-equilibrium state quantified by temperature and composition gradients across the interface.Concerning temperature, to understand how its profile might affect the phase-field simulations, we must first derive theequilibrium assumptions in the Gibbs-Thomson condition.

The Gibbs-Thomson condition in two-dimensions $[6,15,17,20,21]$ relates the temperature at the interface, $T_{i}$, to the deviation from the melting temperature, $T_{m}$, caused by the curvature and velocity of the interface, $T_{i}=T_{m}-\frac{T_{m}}{L} \sum_{i=1,2}\left[\gamma(\hat{n})+\gamma(\hat{n})_{\theta \theta}\right] \frac{1}{R_{i}}-\frac{V_{n}}{\mu(\hat{n})}$, where $L$ is the latent heat, $\gamma(\hat{n})$ is the interfacial free energy, $\theta_{i}$ are the local angles between the normal direction $n$, and the two principle directions and $R_{i}$ is the principle radii of curvature. The final two terms, $V$ and $\mu$, are the normal velocity of the interface and kinetic coefficient respectively. The GibbsThomson condition defines the interfacial stiffness, $\gamma(\hat{n})+\gamma(\hat{n})_{\theta \theta}$, in equilibrium where it is independent of thermal gradients. However, recent studies of solid-solid phase interactions using the capillary fluctuation method[22, 23] have shown that the interfacial stiffness is indeed dependent on the thermal conditions. In rapid solidification processes, where temperature gradient and interface velocity define the microstructure, it is necessary to define an interfacial stiffness in terms of both normal orientation and temperature gradient.

In this paper, we use MD simulations to apply non-equilibrium thermal conditions through temperature gradient profiles perpendicular to the interface and calculate the interfacial free energies, stiffness and anisotropic characteristics for a pure aluminum structure.These interfaceproperties, calculated through the capillary fluctuation method CFM[24], are compared to a thermal equilibrium case when the gradient is zero and the structure temperature is set to the melting temperature. From the data, we candevelop material specifictemperature gradientdependentrelationships for interfacial stiffness and free energy. These properties can be used in subsequent multi-scale computational studies where interfacial stiffness and energy are key parameters.

\section{Theory}

There have been various methods developed to study interfacial properties in molecular dynamic (MD) simulations. For solid-liquid solidification systems in equilibrium, the interfacial stiffnessis often calculated using the capillary fluctuation method (CFM)[25-27] on a thin quasi$2 \mathrm{D}$ system. This method relatesthe interfacial stiffness to the mean square amplitude, $h(x)=$ $\sum_{k} A(k) e^{i k x}$, through equipartition and Fourier mode analysis such that:

$$
\left\langle|A(k)|^{2}\right\rangle=\frac{k_{b} T_{\sigma}}{b W(\mathrm{~S}(\theta)) k^{2}}
$$

where $k_{b}$ is the Boltzmann constant, $T_{\sigma}$ is the temperature of the system, $W$ is the width, $b$ is the thickness, $k$ is the wavenumber, $\gamma$ represents the interface free energy and $\theta$ if the angle between 
the interface normal and the nominally flat normal direction. As with the Gibbs-Thomson condition, theinterfacial stiffness, defined here as $S(\theta)=\gamma+\gamma_{\theta \theta}$, inherently implies equilibrium across the interface. To introduce a temperature dependent term to the CFM, we must first look at the derivation of the system energy.

Hoyt[26] expressed the excess free energy of the two-phase system in equilibrium as $E=$ $b \int_{0}^{W} \gamma(\theta) d s$. At the atomic scale for short time steps, the interface fluctuations are small in amplitude such that $\sqrt{1+h^{\prime}(x)^{2}} \approx 1+\frac{h^{\prime}(x)^{2}}{2}$ and $h^{\prime}(x)=\tan (\theta) \approx \theta$. We can therefore redefine the energy equation with potential contributions from the temperature gradient, $\mathrm{G}$ as:

$$
E=b \int_{0}^{W} \gamma(\theta, \mathrm{G}) \sqrt{1+h^{\prime}(x)^{2}} d x
$$

To include the effects of a one-dimensional temperature gradient, or $G=\nabla T=\frac{d T}{d h}$, on the interfacial free energy, $\gamma$, we modify Hoyt's methodology to expand the multi-variable expression through a second-order Taylor series:

$$
\gamma(\theta, \mathrm{G}) \approx \gamma+\gamma_{\theta} \theta+\gamma_{G} G+\frac{1}{2}\left(\gamma_{\theta \theta} \theta^{2}+\gamma_{G G} G^{2}\right)+\gamma_{\theta G} \theta G
$$

By substituting Equation ( 3 ) into Equation ( 2 ), we can separate the energy equation into the summation of five separate contributions along the interface, Table 1:

Under periodic boundary conditions parallel to the interface length we can reduce the second contribution term to zero, $\int_{0}^{W} h^{\prime}(x) d x=0$. Similarly under the assumption of small height fluctuations, the higher order terms of $h^{\prime}(x) \approx 0$, reducing the fourth and fifth energy contributions to zero. The equation can be further simplified if we consider the first contribution to be the energy of a flat interface and define $\Delta E=E-E_{1}$ :

$$
\Delta E=\frac{1}{2} b\left(\gamma+\gamma_{\theta \theta}+\gamma_{G} G+\frac{\gamma_{G G} G^{2}}{2}\right) \int_{0}^{W} h^{\prime}(x)^{2} d x
$$

If we compare Equation ( 4 ) to Hoyt's derivation for a system in equilibrium[26], $\Delta E=$ $\frac{1}{2} b\left(\gamma+\gamma_{\theta \theta}\right) \int_{0}^{W} h^{\prime}(x)^{2} d x$, we notice the equations are identical when the gradient is zero and that stiffness can be defined by the term within the parenthesis:

$$
S_{(\theta, G)}=\gamma+\gamma_{\theta \theta}+\gamma_{G} G+\frac{1}{2} \gamma_{G G} G^{2}
$$

By taking the second order expansion of the Taylor series for the interfacial free energy, an initial hypothesis is inferred that the stiffness has a power relationship with the gradient. It is possible that this relationship will be material-dependent, but will not be explored in this work. The new derivation of stiffness satisfies the boundary conditions for the equilibrium state when there is no thermal gradient, i.e. $S_{(\theta, \mathrm{G}=0)}=\gamma+\gamma_{\theta \theta}$. By substituting the derived gradient-based interfacial stiffness intoEquation ( 1 ), we can approximate a CFM equation which includes an applied temperature gradient:

$$
\left\langle|A(k)|^{2}\right\rangle=\frac{k_{b} T_{\sigma}}{b W\left(\gamma+\gamma_{\theta \theta}+\gamma_{G} G+\frac{1}{2} \gamma_{G G} G^{2}\right) k^{2}}
$$


However, this derivation assumes that all of the height fluctuations are caused by the change in interfacial stiffness which may be an oversimplification for systems in rapid solidification. From Karma's work on fluctuations in solidifications[11], we know that there are additional contributions to these fluctuations that are independent of stiffness. This was shown by deriving an equation for the fluctuation spectrum for a stationary interface in a temperature gradient:

$$
\left\langle\xi_{k} \xi_{-k}\right\rangle_{G}=\frac{k_{b} T_{\sigma}}{\gamma\left(k^{2}+a^{-2}\right)}
$$

where $\left\langle\xi_{k} \xi_{-k}\right\rangle_{G}$ is defined as the fluctuation spectra of a symmetric structure with a temperature gradient, $a=\sqrt{\gamma T_{\sigma} / L G}$, and $L$ is equal to latent heat per unit volume. If we incorporate the quasi two-dimensional formulation of Equations ( 5 ) and ( 6 ), we can derive a solvable equation for the gradient-dependent stiffness term:

$$
\left\langle|A(k)|^{2}\right\rangle=\frac{k_{b} T_{\sigma}}{b W S_{(\theta, G)}\left(k^{2}+a^{-2}\right)}
$$

where $\gamma$ in the $a$ term is the surface energy under equilibrium conditions. We can then calculate stiffness by plotting $\frac{k_{b} T_{\sigma}}{b W\left\langle|A(k)|^{2}\right\rangle}$ versus $\left(k^{2}+a^{-2}\right)$ and taking the linear slot for low values of wavenumbers.

\section{Methods}

In this section, we define the various MD parameters and methods used in this work to calculate the interface characteristics. This includes the dimensions of the simulation model, method used to develop a two-phase state, applications of a thermal gradient, identification of the solid-liquid interface and finally, the application of the CFM to extract the interface properties.

\subsection{Simulation Structure Dimensions}

The interfacial free energy of aluminum is calculated using LAMMPS molecular dynamics software[28]. The geometry of the aluminum samples used for the MD simulations varies based on the lattice orientation. However, each structure meets the CFMassumptions of $b<<$ $W$ andthe height of the structures are defined as twice the width, $H=2 W$, to limitthe effects between the two interfaces and boundaries,Figure 1.As a result, the structure for each interface orientation varies in dimensions and numbers of atoms, Table 2.

An embedded-atom method (EAM) potential[29], intended forAlCu alloys, is used for the aluminum structures evaluated in this paper. TheEAM potential was selected for future inclusions of copper alloy in similar calculations where the free energy may also be a function of the alloyed concentration in addition to the gradient. Unlike the more recent EAM potentials developed by Mendelev et al.[30] for theindividual application of aluminum or copper, this alloy-based EAM potential was not calibrated to high temperature applications. As a result, the computed melting temperature of $750 \mathrm{~K}$ is much less than the expected value of $933 \mathrm{~K}$ [31]. However, the results presented in this paper focus on the change in results of non-equilibrium conditions relative to the zero-gradient equilibrium results. 


\subsection{Solid-Liquid Phase Structure}

There are numerousmethodsthat can create a two phase solid-liquid MD model. The oneused in this work, first equilibrates the structure to the estimated melting temperature using the NVE ensemble with a fix Berendsen barostat to apply a zero external pressure condition. The temperature parameter is applied through a full-structure Langevin thermostat as the approximate melting temperature. After equilibrating for $20 \mathrm{ps}$ with a time step of $2 \mathrm{fs}$, a new thermostat is defined in the desired liquid portion of the structure, and the temperature is first increased by $500 \mathrm{~K}$ for $50 \mathrm{ps}$ and then cooled back to the melting temperature. The temperature, applied through the full structure thermostat, is held until an equilibrated state is reached, approximately $1 \mathrm{~ns}$ in these simulations. During the equilibration step, the interface between solid and liquid phases is observed to verify no translational movement and that the assumed melting temperature is accurate. With the melting temperature verified, the Langevin thermostat is removed and the structure is equilibrated first using only the Berendsen barostat for 200ps. Finally, the pressure barostat is removed, leaving only the NVE ensemble, and is run for an additional 200ps. Thismethod creates a stable solid-liquid interface under thermal equilibrium conditions, Figure 2, for general applications of material selection.

\subsection{Application of Thermal Gradients}

With a stable two-phase structure in thermal equilibrium at the melting temperature, we can define a method to apply thermal gradients while maintaining a stationary interface. The method devised for this work applies a Langevin thermostat, 20 angstroms thick, to the center of each phase, with an NVE ensemble applied to the remaining portions of the model, Figure 3. These thermostats are defined as dynamic regions, meaning the atoms in the system are allowed to move freely in and out of the area without restrictions. The size of the thermostats is kept to a small portion of the model to reduce any unwanted influence on the interface, verified through the post processed temperature profile at the end of the equilibration.After ramping the Langevin thermostats to the desired temperatures over 200ps, and with an applied zero external pressure boundary condition, the simulation is again equilibrated with the new temperature profile for an additional 200ps. Finally, the simulation is run for an additional 400ps with a snapshot of the atom positions at every $0.2 \mathrm{ps}$ resulting in 1,000 snapshots of the atomic structure, used for the application of the CFM.

To calculate what temperatures the thermostats should be to get the desired temperature gradients, it is helpful to look at the application of Fourier's law for the conservation of heat flux across the interface:

$$
\frac{1}{z_{L}} \int_{T_{m}}^{T_{L}} \kappa_{L} d T=\frac{2}{z_{S}} \int_{T_{S}}^{T_{m}} \kappa_{S} d T
$$

While we are working with a constant material for both phases, the use of a temperature gradient as well as two phases results in a non-linear conductivity, $\kappa(T)$, along the temperature profile, Figure 3. In MD simulations, the thermal conductivity can be calculated for a material given a specific temperature by first calculating the heat flux of the system and then relating it to the conductivity term through the Green-Kubo formulas. This method was used to create two functions, $\kappa_{L}$ and $\kappa_{S}$, for the two phases of the structure. Then, using the location of the interfaces at equilibrium, we can define a desired temperature gradient and calculate the 
temperatures for the two thermostats. In this paper, we are interested in a range of gradients, approximately from $11 \sim 35 \mathrm{~K} / \mathrm{nm}$, to develop a trend in the interfacial characteristics. Since the dimensions of the structures vary based on the interface orientation, Table 2, to maintain the comparable gradients, the calculation of thermostat temperatures must account variations in height and location of the equilibrium interface. The resulting calculations of thermostat temperatures have been provided in Table 3 for reference.

\subsection{Defining the Solid-Liquid Interface}

To identify the location of the interface, a centrosymmetry parameter for FCC structures, $P=\sum_{i=1}^{6}\left|\vec{r}_{i}+\vec{r}_{i+6}\right|^{2}$, is assigned for each atom, $i$, where $\vec{r}$ is used to indicate the vector position of the nearest neighbors.During the simulation, each structure is partitioned into a mesh for collecting the mean centrosymmetry parameter per bin. The size of the bins along the $\mathrm{x}$-axis contribute to how detailed the interface is defined. For consistency across the model sizes, the bin size $(\mathrm{X} \times \mathrm{Y})$ defined for this work is $4.050 \times 2.025$ angstroms. With the average parameter calculated, we can create height profiles perpendicular to the interface and plotthe results of the mean centrosymmetry parameters per bin, Figure 4 .We define the interface as the maximum difference in centrosymmetry between a region above and below the specified height point. This process is repeated along the interface length to develop an interface height profile, $h(x)$, for each snapshot, which is then used for the CFM.

\subsection{CFM and Anisotropy}

Through the derivation outlined in Section 2 of this paper, we know that interfacial stiffness can be connected to the fluctuation in interface height through Equations( 5 ) and ( 6 ) where $h(x)=\sum_{k} A(k) e^{i k x}$. The interfacial stiffness can then be extracted from a linearly proportional relationship between $\frac{k_{b} T_{\sigma}}{b W\left\langle|A(k)|^{2}\right\rangle}$ versus $\left(k^{2}+a^{-2}\right)$. In addition, Hoyt et. al.[25] have shown for the weakly anisotropic crystal nature of solid-liquid interfaces, the interfacial free energy can be obtained as a function of the interface normal, $\gamma(\hat{n})$. This term can be expanded through cubic harmonics[27, 31, 32] such that:

$$
\frac{\gamma(\hat{n})}{\gamma_{0}}=1+\delta_{1}\left(\sum_{i=1}^{3} n_{i}{ }^{4}-\frac{3}{5}\right)+\delta_{2}\left(3 \sum_{i=1}^{3} n_{i}{ }^{6}+66 n_{1}{ }^{2} n_{2}{ }^{2} n_{3}{ }^{2}-\frac{17}{7}\right)
$$

where $n_{i}$ are the components of the interface normal $\hat{n}$ in the Cartesian coordinate system $\left(x_{1}\right.$, $\left.x_{2}, x_{3}\right) . \delta_{1}$ and $\delta_{2}$ are related to the parameters for surface anisotropy. For quasi-2D structures, where the thickness is negligible, the interface normal can be defined as:

$$
\begin{gathered}
n_{i}=\left[\sin (\theta) \hat{x}_{1}+\cos (\theta) \hat{x}_{3}\right] \hat{z}_{i}, \quad i=1,2,3 \\
\hat{x}_{i}=\frac{a_{i} \hat{z}_{1}+b_{i} \hat{z}_{2}+c_{i} \hat{z}_{3}}{\sqrt{a_{i}{ }^{2}+b_{i}{ }^{2}+c_{i}{ }^{2}}}
\end{gathered}
$$

where $\hat{z}_{i}$ is equal the unit vectors [100], [010], and [001] and the $a_{i}, b_{i}, c_{i}$ terms are used to define the interface orientation. Under the assumption that the anisotropic characteristics of the solid-liquid interface are independent of thermal conditions, we can derive equations for the various orientations at equilibrium that satisfy $\gamma+\left.\gamma_{\theta \theta}\right|_{\theta=0}$, Table 4.Theoretically, with the 
selection of three discrete orientations, we can calculate an interfacial energy along with the $\epsilon$ and $\delta$ anisotropic parameters. For verification, we look at a total of six interface orientations. In the following section, we calculate the interface material properties for an aluminum solid-liquid interface in thermal non-equilibrium and develop a trend for use in interpolation. In addition to the calculation of interfacial stiffness and energy, we test the hypothesis that the anisotropy parameters, $\delta_{1}$ and $\delta_{2}$, are independent variables of the applied gradient.

\section{Results and Discussion}

In this section, the interfacial stiffness of aluminum is calculated for six lattice configurations, Table 2, and used to determine the anisotropic characteristics. More specifically, the solid-liquid MD simulations are initially run at the melting temperature, and then with approximate gradients of 12,23 and $34 \mathrm{~K} / \mathrm{nm}$ to identify gradient-dependent behaviors. An interfacial energy per temperature gradient relationship is identified and verified against the presented theory in Section 2. Finally, we check the assumption that material anisotropy is independent of the gradient by calculating the interfacial energy using the equilibrium anisotropy parameters compared to the temperature gradient specific parameters.

As a control, the interfacial stiffness is initially calculated at thermal equilibrium when the temperature of the system is set to the melting temperature for the EAM potential, $750 \mathrm{~K}$, for aluminum. The latent heat per unit volume, $L$, used for aluminum is approximated as $867 \frac{\mathrm{mJ}}{\mathrm{cm}^{3}}$ and is used to calculate the stiffness-independent fluctuation contribution, Equation ( 8 ). The stiffness is defined as the average of the two interfaces for each orientation.The stiffness values for the $100[010], 110[001]$, and $110[\overline{1} 10]$ orientations are used to calculate interfacial free energy and the anisotropy parameters resulting in: $\gamma_{0}=53.3 \mathrm{~mJ} / \mathrm{m}^{2}, \delta_{1}=5.54 \%$ and $\delta_{2}=$ $-0.10 \%$. The expected interfacial energy of aluminumis in the range of $140 \sim 170 \mathrm{~mJ} / \mathrm{m}^{2}$ based on experimental data and well fitted EAM and MEAM potentials[31]. Given the large discrepancy in melting temperature for the selected potential, as described in Section 3.1, the poor representation of interfacial energy at equilibrium is not surprising. These results highlight the need for controlled simulations to be used for reference in MD simulations. When the parameters are verified against the other three orientations, the stiffness can be predicted within $8 \%$ of the calculated value, which is within the typical spread for this CFM method for results using one random seed number.Following the same procedure, the stiffness is calculated for each temperature gradient in each orientation and the results of the 100[010], 110[112], and $110[\overline{1} 10]$ orientations, Figure 5, are again used to calculate temperature-dependent properties.It should be noted that when $G \neq 0$, the x-intercept should be located at $a^{-2}$ rather than zero, as it is for the standard equilibrium case.

Under the assumption thatthe anisotropy of the solid-liquid interface is independent of thermal gradient, we can use the $\delta_{1}$ and $\delta_{2}$ parameters, calculated under equilibrium conditions, to calculate the interfacial free energy for each orientation using the equations inTable 4.Averaging the interfacial energy of the orientations resultsin a linear interfacial energy-temperature gradient relationship,Figure 6 . To verify the assumption of temperature gradient independence for material anisotropy, we repeat the steps of equilibrium to calculate the gradient-dependent parameters, Figure 7. From the plot of anisotropy parameters, there appears to be no correlation between anisotropy and temperature gradient. To verify, the interfacial energies are calculated using the three orientations and the new gradient-dependent anisotropy, Figure 6. Comparing the 
interfacial energies calculated from the gradient dependent and independent anisotropies, it is confirmed that there is negligible impact due to the applied gradient.

From the plot of interfacial free energy versus temperature-gradient, an energy function for aluminum can be identified as $\gamma_{(\theta, G)}=\mu G+\gamma_{o}$, where $\mu=0.746 \frac{\mathrm{mJ} \cdot \mathrm{nm}}{\mathrm{m}^{2} \mathrm{~K}}$ and $\gamma_{o}$ is equal to the interfacial free energy at thermal equilibrium.Returning to the hypothesis that interfacial stiffness can be derived from a second order Taylor expansion of the interfacial energy, Equation ( 5 ), we see that the higher order term can be dropped in the case of pure aluminum where the energygradient relationship is linear. Therefore, the solid-liquid interfacial stiffness for aluminum can be theoretically defined as $S_{(\theta, G)}=\gamma+\gamma_{\theta \theta}+\mu G$, and compared to the MD simulation calculations, Figure 8. The error bars, shown in the figure, provide the deviation between the interfacial stiffness calculated between the two interfaces in the structure. The MD results show good agreement with the hypothesized theory confirming the assumptions of gradientindependent anisotropy parameters and first order Taylor expansion of interfacial energy. With additional sampling of random seed numbers, the gap between theory and simulations is expected to close further.

\section{Conclusions}

MD simulations are performed for applications in CFM on a solid-liquid interface of pure aluminum. The interfacial properties of energy, stiffness and anisotropy are calculated under thermal equilibrium and non-equilibrium conditions seen in rapid solidification processes, such as additive manufacturing. The application of a temperature gradient across the interface results in increased stiffness and energy properties while retaining anisotropy characteristics. These increased parameters can be theoretically explained through a multi-variable Taylor expansion of interfacial free energy and the derivation of the system energy at the solid-liquid interface. The resulting energy and stiffness equations are $\gamma_{(\theta, G)}=\mu G+\gamma_{o}$ and $S_{(\theta, G)}=\gamma+\gamma_{\theta \theta}+\mu G$ respectively. For the EAM modeled aluminum presented in this work, $\mu=0.746 \frac{\mathrm{mJ} \cdot \mathrm{mm}}{\mathrm{m}^{2} \mathrm{~K}}$ and $\gamma_{0}=53.3 \mathrm{~mJ} / \mathrm{m}^{2}$. While published sources state typical gradients for additive manufacturing processes to be on the range of $10^{3} \sim 10^{5} \mathrm{~K} / \mathrm{mm}[12,19]$, these numbers are estimated at larger scales through finite element analysis(FEA) approaches and may not accurately represent the gradient across the interface at an atomic scale. MD simulations are limited in size to a length scale on the order of nanometers which in turn limits the applied temperature gradient. Regardless, the work presented develops a temperature gradient-stiffness and energy relationship which can be interpolated for additional computational applications.

The results conclude that the solid-liquid interface properties can be influenced by a temperature gradient across the interface, replicating a rapid solidification system. Consequently, the dendrite growth phase-field simulations that rely on the application of the Gibbs-Thomson condition to determine the temperature at the interface, may be inaccurately assuming equilibrium conditions for systems of extreme heating and cooling rates.As a result, it may be a contributing factor to the poor phase-field geometric representations at high interface velocities. By incorporating the non-equilibrium thermal conditions into the multi-scale computational methods, we can begin to close the gap between experimental inconsistency in material strength and the predicted computational results. Additional factors remaining and the future work 
includes a correlation study of: the temperature at the interface deviating from the melting temperature and the composition concentration defined in metal-alloys in connection with the temperature gradient studied in this work.

\section{Acknowledgements}

N.B. acknowledges the support of the US Department of Energy (DOE) through the AAPP Program for this work. This research used resources provided by the LANL Institutional Computing Program. LANL, an affirmative action/equal opportunity employer, is operated by Los Alamos National Security, LLC, for the National Nuclear Security Administration of the U.S. DOE under contract DE-AC52-06NA25396.N.B.would also like to acknowledge the support of the National Science Foundation(NSF) through CMMI 1624313.

\section{References}

[1] D. Gu, W. Meiners, K. Wissenbach, R. Poprawe, Laser additive manufacturing of metallic components: materials, processes and mechanisms, International materials reviews 57(3) (2012) 133-164.

[2] R.R. Dehoff, M.M. Kirka, F. List, K.A. Unocic, W.J. Sames, Crystallographic texture engineering through novel melt strategies via electron beam melting: Inconel 718, Materials Science and Technology 31(8) (2015) 939-944.

[3] C.-M. Liu, H.-M. Wang, X.-J. Tian, H.-B. Tang, D. Liu, Microstructure and tensile properties of laser melting deposited $\mathrm{Ti}-5 \mathrm{Al}-5 \mathrm{Mo}-5 \mathrm{~V}-1 \mathrm{Cr}-1 \mathrm{Fe}$ near $\beta$ titanium alloy, Materials Science and Engineering: A 586 (2013) 323-329.

[4] E.R. Denlinger, J.C. Heigel, P. Michaleris, T. Palmer, Effect of inter-layer dwell time on distortion and residual stress in additive manufacturing of titanium and nickel alloys, Journal of Materials Processing Technology 215 (2015) 123-131.

[5] J. Hoyt, M. Asta, A. Karma, Atomistic and continuum modeling of dendritic solidification, Materials Science and Engineering: R: Reports 41(6) (2003) 121-163.

[6] D. Tourret, A.J. Clarke, S.D. Imhoff, P.J. Gibbs, J.W. Gibbs, A. Karma, Three-dimensional multiscale modeling of dendritic spacing selection during Al-Si directional solidification, JOM 67(8) (2015) 1776-1785.

[7] I.A. Roberts, C.J. Wang, R. Esterlein, M. Stanford, D.J. Mynors, A three-dimensional finite element analysis of the temperature field during laser melting of metal powders in additive layer manufacturing, International Journal of Machine Tools and Manufacture 49(12) (2009) 916-923.

[8] I. Steinbach, L. Zhang, M. Plapp, Phase-field model with finite interface dissipation, Acta Materialia 60(6) (2012) 2689-2701.

[9] H. Wang, W. Kuang, X. Zhang, F. Liu, A hyperbolic phase-field model for rapid solidification of a binary alloy, Journal of Materials Science 50(3) (2015) 1277-1286.

[10] D.M. Herlach, Non-equilibrium solidification of undercooled metallic metls, Materials Science and Engineering: R: Reports 12(4) (1994) 177-272.

[11] A. Karma, Fluctuations in solidification, Physical Review E 48(5) (1993) 3441.

[12] C. Levi, R. Mehrabian, Heat flow during rapid solidification of undercooled metal droplets, Metallurgical Transactions A 13(2) (1982) 221-234.

[13] R. Willnecker, D. Herlach, B. Feuerbacher, Evidence of nonequilibrium processes in rapid solidification of undercooled metals, Physical review letters 62(23) (1989) 2707. 
[14] M. Aziz, Model for solute redistribution during rapid solidification, Journal of Applied Physics 53(2) (1982) 1158-1168.

[15] P. Nie, O. Ojo, Z. Li, Numerical modeling of microstructure evolution during laser additive manufacturing of a nickel-based superalloy, Acta Materialia 77 (2014) 85-95.

[16] L.-Q. Chen, Phase-field models for microstructure evolution, Annual review of materials research 32(1) (2002) 113-140.

[17] P. Collins, D. Brice, P. Samimi, I. Ghamarian, H. Fraser, Microstructural Control of Additively Manufactured Metallic Materials, Annual Review of Materials Research (0) (2016).

[18] I. Beyerlein, M. Demkowicz, A. Misra, B. Uberuaga, Defect-interface interactions, Progress in Materials Science 74 (2015) 125-210.

[19] I. Gibson, D.W. Rosen, B. Stucker, Additive manufacturing technologies, Springer2010.

[20] J.T. McKeown, K. Zweiacker, C. Liu, D.R. Coughlin, A.J. Clarke, J.K. Baldwin, J.W. Gibbs, J.D. Roehling, S.D. Imhoff, P.J. Gibbs, Time-Resolved In Situ Measurements During Rapid Alloy Solidification: Experimental Insight for Additive Manufacturing, JOM 68(3) (2016) 985-999.

[21] A. Karma, D. Tourret, Atomistic to continuum modeling of solidification microstructures, Current Opinion in Solid State and Materials Science 20(1) (2016) 25-36.

[22] Y. Mishin, Atomistic modeling of the $\gamma$ and $\gamma^{\prime}$-phases of the Ni-Al system, Acta Materialia 52(6) (2004) 1451-1467.

[23] B. Cheng, G.A. Tribello, M. Ceriotti, Solid-liquid interfacial free energy out of equilibrium, Physical Review B 92(18) (2015) 180102.

[24] R.L. Davidchack, B.B. Laird, Direct calculation of the crystal-melt interfacial free energies for continuous potentials: Application to the Lennard-Jones system, The Journal of chemical physics 118(16) (2003) 7651-7657.

[25] J. Hoyt, M. Asta, A. Karma, Method for computing the anisotropy of the solid-liquid interfacial free energy, Physical Review Letters 86(24) (2001) 5530.

[26] J. Hoyt, Z. Trautt, M. Upmanyu, Fluctuations in molecular dynamics simulations, Mathematics and Computers in Simulation 80(7) (2010) 1382-1392.

[27] R.L. Davidchack, J.R. Morris, B.B. Laird, The anisotropic hard-sphere crystal-melt interfacial free energy from fluctuations, The Journal of chemical physics 125(9) (2006) 094710.

[28] S. Plimpton, Fast parallel algorithms for short-range molecular dynamics, Journal of computational physics 117(1) (1995) 1-19.

[29] J. Cai, Y. Ye, Simple analytical embedded-atom-potential model including a long-range force for fcc metals and their alloys, Physical Review B 54(12) (1996) 8398.

[30] M. Mendelev, M. Kramer, C.A. Becker, M. Asta, Analysis of semi-empirical interatomic potentials appropriate for simulation of crystalline and liquid $\mathrm{Al}$ and $\mathrm{Cu}$, Philosophical Magazine 88(12) (2008) 1723-1750.

[31] E. Asadi, M.A. Zaeem, S. Nouranian, M.I. Baskes, Two-phase solid-liquid coexistence of $\mathrm{Ni}, \mathrm{Cu}$, and $\mathrm{Al}$ by molecular dynamics simulations using the modified embedded-atom method, Acta Materialia 86 (2015) 169-181.

[32] W. Fehlner, S. Vosko, A product representation for cubic harmonics and special directions for the determination of the Fermi surface and related properties, Canadian Journal of Physics 54(21) (1976) 2159-2169. 
Interfacial Stiffness and Free Energy Dependent on Temperature Gradient

Liquid Thermostat
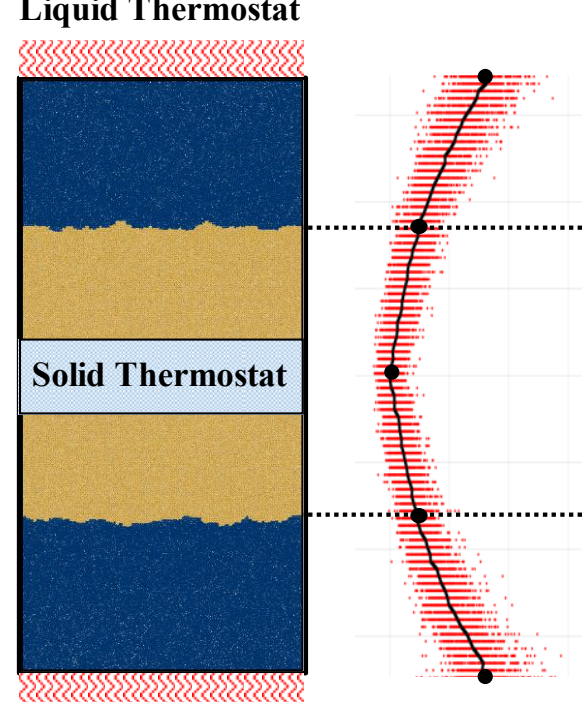

Liquid Thermostat

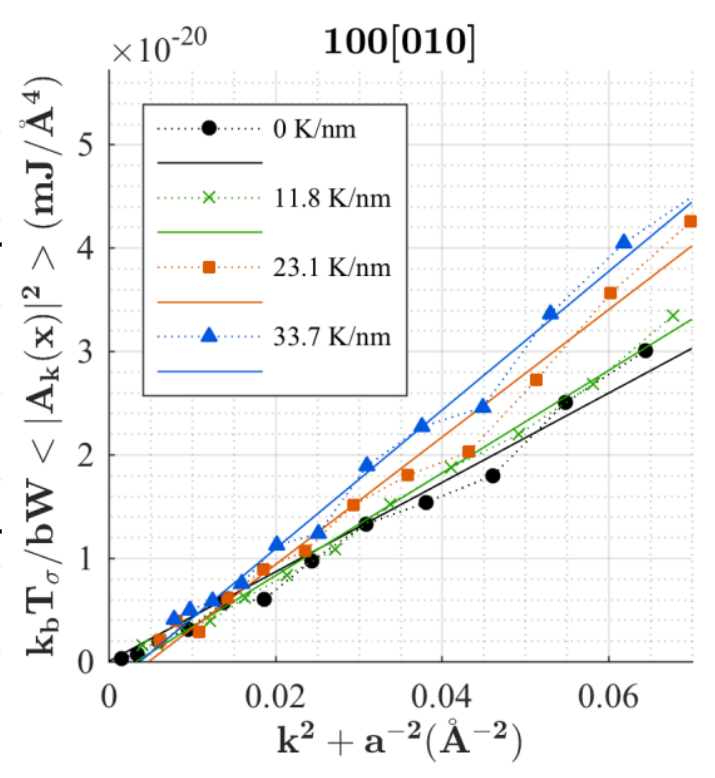


Table 1: Approximate energy contributions at an interface with applied temperature gradient

\begin{tabular}{|l|l|}
\hline$E:$ & $b \int_{0}^{W} \gamma(\theta, \mathrm{G}) \sqrt{1+h^{\prime}(x)^{2}} d x=\sum_{i=1}^{5} E_{i}$ \\
\hline$E_{1}:$ & $b \int_{0}^{W}\left[\gamma+\gamma_{G}+\frac{\gamma_{G G} G^{2}}{2}\right] d x$ \\
\hline$E_{2}:$ & $b \int_{0}^{W} h^{\prime}(x)\left[\gamma_{\theta}+\gamma_{\theta G} G\right] d x=0$ \\
\hline$E_{3}:$ & $b \int_{0}^{W} h^{\prime}(x)^{2}\left[\frac{\gamma}{2}+\frac{\gamma_{\theta \theta}}{2}+\frac{\gamma_{G} G}{2}+\frac{\gamma_{G G} G^{2}}{4}\right] d x$ \\
\hline$E_{4}:$ & $b \int_{0}^{W} h^{\prime}(x)^{3}\left[\frac{\gamma_{\theta}}{2}+\frac{\gamma_{\theta G} G}{2}\right] d x=0$ \\
\hline$E_{5}:$ & $b \int_{0}^{W} h^{\prime}(x)^{4}\left[\frac{\gamma_{\theta \theta}}{4}\right] d x=0$ \\
\hline
\end{tabular}


Table 2: Simulation sizes for six interface orientations

\begin{tabular}{|c|c|c|c|c|}
\hline Orientation* & Atoms & $\mathbf{H ~}(\AA)$ & $\mathbf{W}(\boldsymbol{\AA})$ & $\mathbf{b}(\AA)$ \\
\hline $100[010]$ & 204,800 & 680 & 332 & 17 \\
\hline $100[012]$ & 460,800 & 682 & 446 & 28 \\
\hline $110[001]$ & 409,600 & 963 & 333 & 23 \\
\hline $110[1 \overline{1} 0]$ & 409,600 & 962 & 469 & 17 \\
\hline $110[1 \overline{1} 2]$ & 460,800 & 723 & 407 & 29 \\
\hline $110[\overline{1} 10]$ & 409,600 & 962 & 469 & 17 \\
\hline
\end{tabular}

*The first number of the notation indicates the direction perpendicular to the interface, the number in brackets denotes the direction along the interface length. 
Table 3: Temperature gradient and thermostat definitions.

\begin{tabular}{|c|c|c|c|c|}
\hline Orientation & $\begin{array}{c}\mathbf{G} \\
(\mathbf{K} / \mathbf{n m})\end{array}$ & $\begin{array}{r}\mathbf{T}_{\mathbf{L}} \\
(\mathbf{K}) \\
\end{array}$ & $\begin{array}{r}\mathbf{T}_{\mathbf{S}} \\
(\mathbf{K}) \\
\end{array}$ & $\begin{array}{l}\mathbf{T}_{\sigma}{ }^{*} \\
(\mathbf{K}) \\
\end{array}$ \\
\hline \multirow{3}{*}{$100[010]$} & 11.8 & 1002 & 594 & 764 \\
\hline & 23.3 & 1308 & 492 & 808 \\
\hline & 34.2 & 1649 & 425 & 871 \\
\hline \multirow{3}{*}{$100[012]$} & 11.8 & 1009 & 600 & 771 \\
\hline & 23.3 & 1320 & 502 & 818 \\
\hline & 34.2 & 1665 & 438 & 882 \\
\hline \multirow{3}{*}{$110[001]$} & 11.8 & 1131 & 553 & 782 \\
\hline & 23.0 & 1602 & 446 & 849 \\
\hline & 33.2 & 2118 & 384 & 931 \\
\hline \multirow{3}{*}{$110[1 \overline{1} 0]$} & 11.8 & 1130 & 553 & 780 \\
\hline & 23.0 & 1599 & 445 & 847 \\
\hline & 33.2 & 2113 & 382 & 931 \\
\hline \multirow{3}{*}{$110[1 \overline{1} 2]$} & 11.8 & 1027 & 593 & 774 \\
\hline & 23.2 & 1362 & 494 & 826 \\
\hline & 34.0 & 1731 & 430 & 898 \\
\hline \multirow{3}{*}{$110[\overline{1} 10]$} & 11.8 & 1133 & 556 & 784 \\
\hline & 22.9 & 1603 & 449 & 852 \\
\hline & 33.2 & 2118 & 387 & 935 \\
\hline
\end{tabular}


Table 4: Anisotropy for multiple interface orientations

\begin{tabular}{|c||c|}
\hline Orientation & $\frac{\boldsymbol{\gamma}+\left.\boldsymbol{\gamma}_{\boldsymbol{\theta} \boldsymbol{\theta}}\right|_{\boldsymbol{\theta}=\mathbf{0}}}{\gamma_{\boldsymbol{0}}}$ \\
\hline \hline $100[010]$ & $1-\frac{18}{5} \delta_{1}-\frac{80}{7} \delta_{2}$ \\
\hline $100[012]$ & $1-\frac{18}{5} \delta_{1}-\frac{80}{7} \delta_{2}$ \\
\hline $110[001]$ & $1-\frac{21}{10} \delta_{1}+\frac{365}{14} \delta_{2}$ \\
\hline $110[1 \overline{1} 0]$ & $1-\frac{39}{10} \delta_{1}+\frac{155}{14} \delta_{2}$ \\
\hline $110[1 \overline{1} 2]$ & $1-\frac{1}{10} \delta_{1}+\frac{295}{14} \delta_{2}$ \\
\hline $110[\overline{1} 10]$ & $1-\frac{39}{10} \delta_{1}+\frac{155}{14} \delta_{2}$ \\
\hline
\end{tabular}




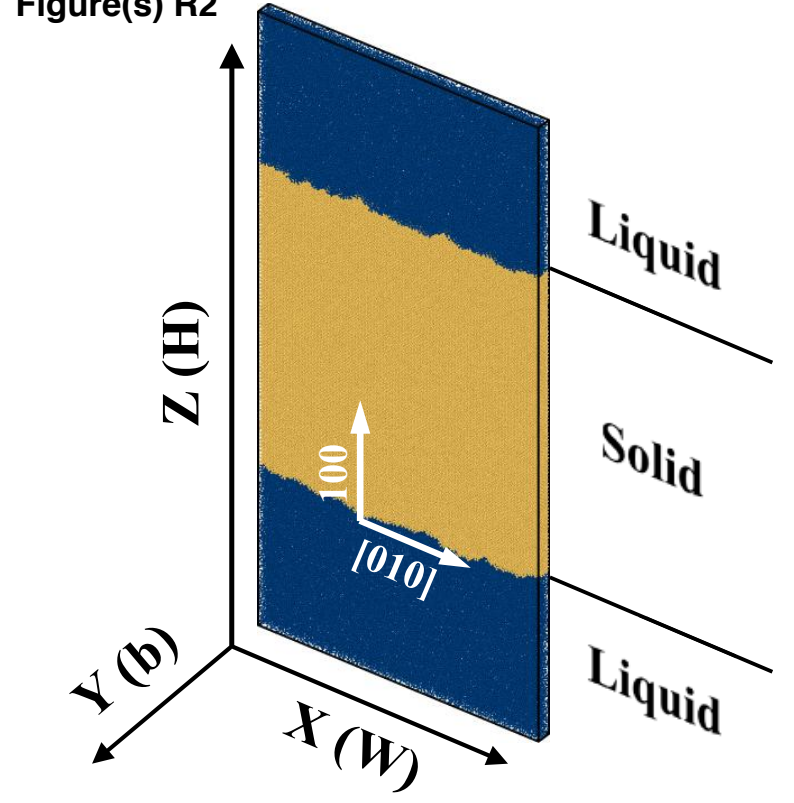

Figure 1. Quasi-2D model of $100[010]$ orientation of solid-liquid simulation model for pure aluminum 


\section{Amorphous Liquid}

\section{Crystalline Solid}

Figure 2. Atomic structure of a system in a two-phase state consisting of an amorphous liquid and crystalline solid. Interface graphically defined as the boundary between the two phases. 
Liquid Thermostat

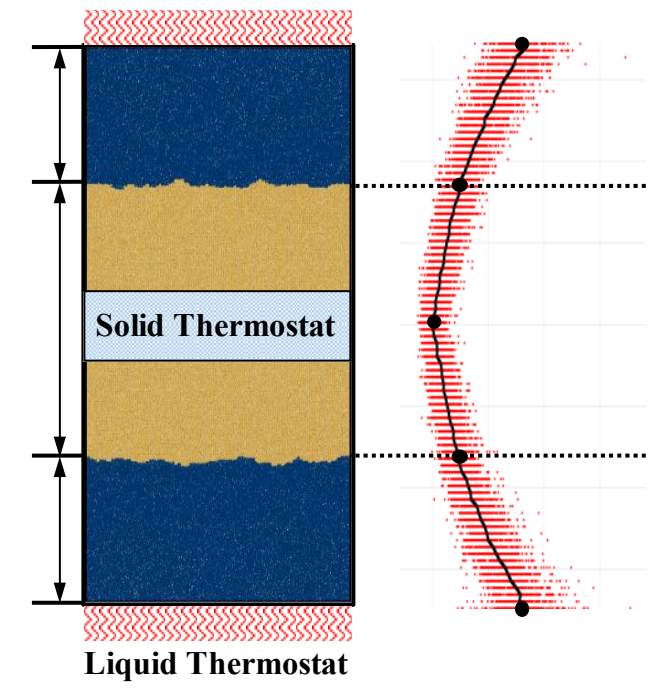

Figure 3. Applied thermostats to a solid-liquid structure of aluminum. Temperature is sampled along the width of the structure for every height and averaged to verify temperature profile. 


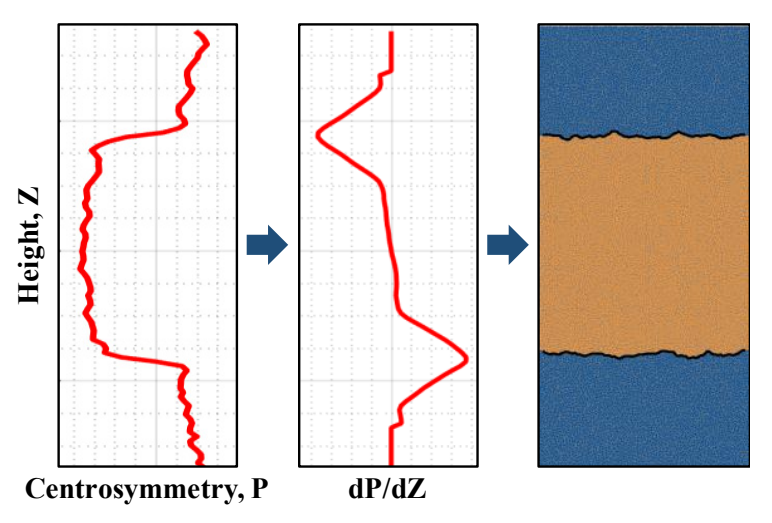

Figure 4. Atomic structure of a structure in a two-phase state consisting of an amorphous liquid and crystalline solid. Interface graphically defined as the boundary between the two phases. 


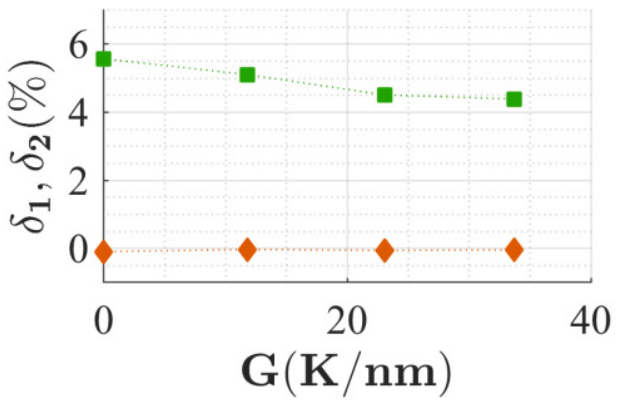

Figure 7. Temperature gradient versus anisotropy parameters for aluminum. 


\section{$110[\overline{1} 10]$}

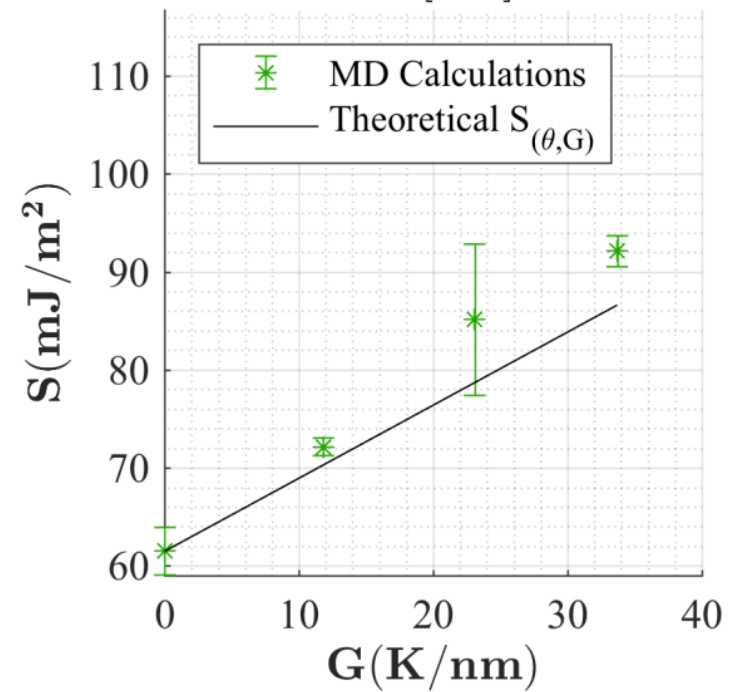

Figure 8. Interfacial stiffness of $110[\overline{1} 10]$ interface orientation. Calculated MD results compared to theoretical equation. 